\title{
Obere Einflussstauung - Vena-cava-superior-Syndrom
}

Nater, Harald ; Niggemeier, Verena ; Stahel, Rolf A ; Franzen, Daniel

DOI: https://doi.org/10.1024/1661-8157/a001306

Other titles: In Process Citation

Posted at the Zurich Open Repository and Archive, University of Zurich ZORA URL: https://doi.org/10.5167/uzh-84430

Journal Article

Accepted Version

Originally published at:

Nater, Harald; Niggemeier, Verena; Stahel, Rolf A; Franzen, Daniel (2013). Obere Einflussstauung Vena-cava-superior-Syndrom. Praxis, 102(9):503-512.

DOI: https://doi.org/10.1024/1661-8157/a001306 


\section{Obere Einflussstauung - Vena Cava Superior Syndrom}

\section{Definition / Leitsymptome}

Das Vena cava superior Syndrom (VCSS) bezeichnet einen Symptomenkomplex, welcher aus einer venösen Abflussbehinderung der Vena cava superior (VCS) resultiert. Dies kann im Rahmen einer Kompression des Gefäßes von aussen oder einer Invasion durch pathologische Veränderungen (i.R. Tumoren) der rechten Lunge oder der mediastinalen Strukturen auftreten. Zusätzlich ist eine Thrombosierung innerhalb der Vena cava superior als Ursache (alleinig oder zusammen mit einer Kompression von aussen) möglich (intraluminale Obstruktion).

Das VCSS trat vor der Antibiotika-Ära häufig in Folge unterschiedlichster unbehandelter Infektionskrankheiten wie z.B. der Tuberkulose oder beim luetischen Aortenaneurysma auf. Heutzutage entsteht das Syndrom in 90\% der Fälle durch ein malignes Geschehen. Im Vordergrund steht hier mit etwa 70\% das Bronchialkarzinom, desweiteren kommen Lymphknotenmetastasen oder mediastinale Raumforderungen in Betracht. Ein nicht zu vernachlässigender Teil, welcher in den letzten Jahren durch die vermehrte Anwendung von zentralvenösen Kathetern und PacemakerElektroden zugenommen hat, ist auf eine Thrombosierung zurück zu führen. Das Ausmass der klinischen Symptome ist davon abhängig, wie schnell es zu einer Obstruktion der VCS kommt und wie schnell Kollateralgefässe entstehen können, welche den venösen Rückstrom in den rechten Vorhof gewährleisten. Die Kollateralen werden häufig von der V. azygos, V. mammaria interna, V. thoracica lateralis, V. paraspinosus oder von Ösophagealvenen gebildet. Durch den erhöhten zentralvenösen Druck kommt es zu den typischen Symptomen wie Gesichtsödem, gestauten venösen Gefässen an Hals und Thorax, Armödemen, Zyanose und Gesichtsplethora. Die Symptome zeigen sich im Verlauf unter erhöhtem Gefässdruck mit zunehmender Dilatation der Kollateralen wieder regredient. Trotz der teilweise imponierenden klinischen Symptome sollte eine zeitnahe zytologische oder histologische Diagnostik bei Verdacht auf eine maligne Genese im Vordergrund stehen. Eine notfallmässige Radiotherapie, welche auch in Kombination mit einer Stentimplantation in die VCS durchgeführt werden kann, ist in schweren Fällen mit Atemwegsobstruktion (Stridor!) oder bei Depression des zentralen Nervensystems in Folge eines Hirnödems indiziert. Diese Therapiemassnahme führt zu einer raschen Symptomkontrolle über längere Zeit ohne die weiterführende Diagnostik zu beeinträchtigen. [1, 2]

\section{Im Artikel verwendete Abkürzungen}
CT Computertomographie
VCSS Vena cava Superior-Syndrom
VCS Vena cava superior 


\section{Klinik und Komplikationen}

\section{Klinik}

Die Diagnose des VCSS kann unabhängig von der Ätiologie klinisch gestellt werden. Dyspnoe ist das häufigste primäre Symptom gefolgt von Gesichts- und Halsschwellung, welche beim Vorbeugen oder Flachliegen progredient sein kann. Im frühen Stadium zeigen sich gelegentlich auch Armschwellungen, ein unproduktiver Husten und/oder Brustschmerzen. In schweren Fällen, bei welchen es aufgrund der venösen Rückstauung zu einem (hydrostatischen) Hirnödem kommt, treten Kopfschmerzen, Verwirrtheit und neurologische Symptome bis hin zum Koma auf. In der klinischen Untersuchung manifestieren sich hauptsächlich Gesichtsödeme sowie gestaute Halsund Kollateralvenen am Thorax. Armödeme, Zyanose und Gesichtsplethora sind weniger häufig. In manchen Fällen ist der Pemberton Test positiv. Hierbei führt beim aufrecht sitzenden Patienten das Anheben beider Arme über Herzhöhe zur Zunahme des Gesichtsödems und Auftreten einer Zyanose. Die genannten Symptome und klinischen Zeichen zeigen sich häufiger bei malignen als bei infektiösen Erkrankungen. Meistens sind sie aufgrund des raschen Tumorwachstums und der damit verbundenen unzureichenden Kompensation der Kollateralgefässe über mehrere Wochen progredient. Im Gegensatz dazu zeigen sich Infektionen wie die Tuberkulose eher weniger eindrücklich, da die Obstruktion der VCS langsam voranschreitet und somit den Kollateralgefässen genügend Zeit gibt, sich zu entwickeln. In diesem Fall ist auch die Erhöhung des intravasalen Druckes in den suprakardialen Gefässen geringer. Im Allgemeinen kommt es nach Ausbildung einer ausreichenden Kollateralzirkulation zu einer Regredienz der Beschwerden. Die Symptomatik der oberen Einflussstauung hängt somit einerseits von der zugrunde liegenden Ätiologie sowie von der Geschwindigkeit der Lumeneinengung der VCS resp. der Möglichkeit zur ausreichenden Kollateralisation ab.

\section{Vena cava superior Syndrom (Stadien nach Yu et al.) [3]}

\begin{tabular}{|l|l|l|}
\hline Grad & Kategorie & Definition \\
\hline 0 & Asymptomatisch & Radiologisch Obstruktion der Vena Cava superior \\
\hline 1 & Leichtgradig & Oedeme an Kopf und Hals, Zyanose, Plethora \\
\hline 2 & Mittelgradig & $\begin{array}{l}\text { Dysphagie, Husten, Beeinträchtigung der Kiefer- und Lidbewegung } \\
\text { aufgrund von Oedem }\end{array}$ \\
\hline 3 & Schwergradig & $\begin{array}{l}\text { Kopfschmerz, Schwindel (aufgrund von intrakranialer Druckerhöhung), } \\
\text { Larynxödem, hämodynamische Symptome wie Synkope beim } \\
\text { Vornüberbeugen }\end{array}$ \\
\hline 4 & Lebensbedrohlich & $\begin{array}{l}\text { Konfusion oder Somnolenz, signifikantes Larynxoedem (Stridor), } \\
\text { signifikante hämodynamische Beeinträchtigung mit Synkope ohne } \\
\text { Provokation, Hypotonie, Niereninsuffizienz }\end{array}$ \\
\hline 5 & Infaust & Tod \\
\hline
\end{tabular}




\section{Komplikationen}

Gesichts- und Halsödem sind optisch eindrücklich, haben aber klinisch wenig Relevanz. Sobald die Schleimhäute durch das Ödem betroffen sind, kann dies jedoch zur Obstruktion der Nasenpassage und des Larynx mit Dyspnoe, Stridor, Husten, Heiserkeit und Dysphagie führen. In schweren Fällen kann sich ein zerebrales Ödem entwickeln und zu Ischämien oder zur Herniation mit möglicher Todesfolge führen. Hämodynamische Beeinträchtigungen aufgrund des verminderten venösen Rückstroms treten sehr selten auf und werden durch Erhöhung des venösen Druckes und durch Kollateralen kompensiert. 
Abklärungsstrategie

\section{Bildgebung}

Im konventionellen Röntgenbild des Thorax finden sich in bis zu 80\% der Fälle pathologische Veränderungen wie eine Mediastinalverbreiterung und/oder Pleuraergüsse. Eine kontrastmittelverstärkte Computertomographie (CT) ist jedoch die Bildgebung der Wahl. Diese lässt neben der Identifikation der Ursache eine Beurteilung des Ausmasses der Obstruktion sowie der Ausprägung und Anzahl der Kollateralgefässe zu. Der Goldstandard zur Abschätzung der Ausprägung einer Obstruktion ist jedoch die Venographie der beiden oberen Extremitäten, welche im klinischen Alltag nur sehr selten durchgeführt wird. Im Vergleich zum CT kann mit der Venographie die Ausdehnung sowie eine mögliche Thrombosierung besser beurteilt werden. Auch Kollateralgefässe werden besser dargestellt. Bei der Identifikation der Aetiologie des VCSS ist die Venographie jedoch klar dem CT unterlegen [4].

Die Spiral-CT-Phlebographie kombiniert die Vorteile der Venographie und eines CT's, setzt aber eine präzise Durchführung der Kontrastmittelapplikation voraus, um Flussartefakte zu vermeiden. Eine Magnetresonanz-Venographie ist eine Alternative für Patienten mit Kontrastmittelallergie oder bei fehlendem venösem Zugang.

Die Wahl der weiterführenden Bildgebung sollte im Hinblick auf eine Intervention zur Gewinnung von Zell- oder Gewebeproben getroffen werden.

\section{Zytologische/histologische Diagnostik}

$\mathrm{Da}$ in der Bildgebung bereits bei extravasaler Kompression ein erster Verdacht auf eine maligne Ursache gestellt werden kann, hat eine zytologische resp. histologische Identifikation für eine adäquate Therapie höchste Priorität. Minimalinvasiven Verfahren soll dabei der Vorzug geben werden. Dazu gehören eine zytologische Untersuchung des Sputums (bei im CT identifiziertem Lungentumor) oder der Pleuraflüssigkeit sowie eine Feinnadelpunktion von vergrösserten Lymphknoten(paketen). Invasive Vorgehensweisen sind angezeigt, wenn die zuvor beschriebenen Abklärungen keine Diagnose zulassen. Dazu gehören Bronchoskopien, Mediastinoskopien, Thorakoskopien oder Thorakotomien. Mediastinale Interventionen weisen bei Patienten mit einem VCSS ein erhöhtes Blutungsrisiko auf. Zudem kann es aufgrund eines Trachealödems zu einer erschwerten Intubation kommen. Bei einem Perikarderguss besteht das Risiko einer hämodynamischen Beeinträchtigung im Rahmen einer Tamponade.

Bei Hochrisikopatienten ist eine perkutane transthorakale CT-gesteuerte Punktion oder eine endoskopische ultraschallgesteuerte Biopsie eine gute Alternative zu den invasiven Verfahren. Je nach Ausmass der Symptome und des Zustandes des Patienten kann eine Diagnose nicht forciert werden, und es muss eine direkte Therapie mit Stenting und sofortiger Radio- oder Chemotherapie eingeleitet werden. 


\section{Diagnosekriterien / Aetiologie / Differenzialdiagnose}

\section{Aetiologie}

Vor der Einführung der Antibiotika kam es vor allem aufgrund von Infektionskrankheiten zum VCSS.

Als Beispiele sind hier das thorakale Aortenaneurysma im Rahmen einer Syphilis, die fibrosierende Mediastinitis im Rahmen einer Tuberkulose oder einer endemischen Mykose (Histoplasmose) zu nennen. Seit der Möglichkeit einer potenten antibiotischen Therapie dieser Erkrankungen stehen maligne Ursachen im Vordergrund. Mit der Installation von zentralvenösen Kathetern und Pacemakern kommt es auch zu einem erhöhten Thromboserisiko in der VCS.

\section{Differenzialdiagnosen}

Malignome sind in 60-86\% für das VCSS verantwortlich. Das nichtkleinzellige Bronchuskarzinom (insbesondere Plattenepithelkarzinom) ist mit 50\% die häufigste Ursache für das VCSS, gefolgt vom kleinzelligen Lungenkarzinom (25\%) und dem Non-Hodgkin Lymphom (10\%). Thymome, mediastinale Keimzelltumoren, Mesotheliome sowie Metastasen in den mediastinalen Lymphknoten (v.a. beim Mammakarzinom) sind deutlich seltener [5].

2-4\% aller Patienten mit einem Bronchuskarzinom erleiden im Verlauf ihrer Erkrankung ein VCSS. Patienten mit einem kleinzelligen Lungenkarzinom sind häufiger davon betroffen. Die Obstruktion der VCS kann entweder durch Tumorkompression von aussen, durch Invasion des Tumors in das Gefäss oder Kompression durch reaktiv veränderte Lymphknoten erfolgen [6].

Auch beim Non-Hodgkin-Lymphom erleiden 2-4\% der Patienten ein VCSS. Das lymphoblastische und das diffuse grosszellige B-Zell-Lymphom sind dabei am häufigsten betroffen. Das primär mediastinale grosszellige B-Zell-Lymphom, welches mit einer Sklerose der Tumormasse einhergeht, zeichnet sich durch eine rasch progrediente Grössenzunahme im vorderen Mediastinum aus und ist daher häufig mit einem VCSS assoziiert. Auch wenn die Patienten im Verlauf die klinischen Kriterien für ein VCSS nicht erfüllen, so zeigt sich doch häufig (bis zu 80\%) in der CT eine Kompression der VCS. Als weitere Ursache für ein VCSS kommen Thromben differenzialdiagnostisch oder als Kofaktor bei einer Kompression von aussen in Betracht. Sie entstehen meistens aufgrund von zentralvenösen Kathetern oder Pacemaker-Elektroden. In Anbetracht der hohen Verbreitung solcher Installationen ist die Inzidenz einer dadurch provozierten Thrombosierung jedoch gering.

Postaktinisch kann es ebenfalls zu einem VCSS kommen, auch wenn die Bestrahlung bereits Jahre zurück liegt. 
Therapie

Grundsätzlich richtet sich die Therapie des VCSS nach der Ausprägung der Symptome.

Evidenz-basierte Richtlinien für die Behandlung des VCSS existieren nicht. Bei einem

Bronchuskarzinom mit symptomatischer oberer Einflussstauung empfehlen das National

Comprehensive Cancer Network (NCCN) und das American College of Chest Physicians (ACCP) jedoch eine Radiotherapie oder Stent-Einlage.

Früher sah man die obere Einflussstauung als Notfallindikation für eine Radiotherapie, was heute nicht mehr zwingend der Fall ist. Da die Symptome über Wochen oder Monate entstehen, haben sich häufig schon Kollateralgefässe gebildet, welche die obere Einflussstauung teilweise kompensieren. Mit der Therapie zu warten bis eine ausreichende Diagnostik erfolgt ist, gefährdet das Leben der Patienten in der Regel nicht und hat keinen Einfluss auf die Langzeitprognose, solange der klinische Zustand stabil ist. Zudem kann eine voreilige Radiotherapie die histologische Diagnosestellung erschweren oder verunmöglichen. Aus dieser Überlegung ist bei symptomatischen Patienten primär eine Stenteinlage vorzuziehen. Darüber hinaus kann mit der Stenteinlage im Vergleich zur Radiotherapie eine raschere Reduktion der Symptome erreicht werden. Die Erfolgsrate nach einer Cava-Stenteinlage liegt bei 95$100 \%$.

Notfallsituationen, die ein schnelles Handeln erfordern, bestehen bei Patienten, welche sich mit einem Stridor oder Koma präsentieren. In diesen Fällen ist eine Notfallbehandlung mittels Stenteinlage und Radiotherapie angezeigt, um ein respiratorisches Versagen und einen unmittelbar tödlich Ausgang zu vermeiden [7]. Der Stent wird perkutan unter Lokalanästhesie über die V. jugularis, subclavia oder femoralis eingebracht. Vor Einsetzen des Stents kann es erforderlich sein, eine Angioplastie mit Ballonkatheter vorzunehmen.

Nach Stenteinlage wird eine kurzzeitige Antikoagulation empfohlen, wobei jedoch bezüglich Dauer und Art keine Evidenz besteht. Je nach Literaturquelle wird eine Antikoagulation von einem bis neun Monaten empfohlen. Teilweise wird aber auch nur eine Thrombozytenaggregationshemmung durchgeführt.

Zu den frühen Komplikationen nach Stentimplantation gehören Infektionen, pulmonale Embolien, die Migration des Stents, Hämatome an der Insertionsstelle, Blutungen und Perforationen oder die Ruptur der VCS. Später kann es zu Blutungen aufgrund der Antikoagulation kommen oder zu einem RezidivVerschluss. Am häufigsten kommt es zu einem Verschluss durch einen Thrombus oder Invasion des Tumors. Letzteres kann mit einem sekundären Stent gut behandelt werden [4].

Es existieren keine Daten, welche einen Vorteil der Hochlagerung des Oberkörpers gegenüber der Flachlagerung aufzeigen konnten. Theoretisch kann es bei letzterer jedoch aufgrund des verlangsamten Blutrückflusses zum Herzen zu Thromben in den oberen Extremitäten oder zu einer verlangsamten Resorption von Medikamenten nach einer intramuskulären Injektion in die oberen Extremitäten kommen. 
Kortikosteroide können bei steroidsensiblen Malignomen wie Lymphomen und Thymomen hilfreich sein. Steroide werden aufgrund ihrer abschwellenden Wirkung aber auch im Rahmen einer Radiotherapie verabreicht, insbesondere wenn ein Larynxoedem besteht. Ihre Wirksamkeit konnte bisher jedoch in keiner Studie belegt werden.

Diuretika werden ebenfalls eingesetzt, auch wenn in einer retrospektiv durchgeführten Studie kein Überlebensvorteil gefunden werden konnte, auch nicht in Kombination mit einer Steroidtherapie.

Bei einem VCSS in Folge eines Thrombus kann eine lokale Thrombolyse über einen entsprechenden Katheter versucht werden. Entscheidend ist jedoch, dass eine therapeutische Antikoagulation eingeleitet wird. Eine Thrombektomie wird in Ausnahmefällen durchgeführt.

Die Chemotherapie ist die Therapie der Wahl bei Patienten mit kleinzelligem Bronchuskarzinom, NonHodgkin-Lymphom oder einem Teratom. Damit zeigen sich eine rasche Verkleinerung der Tumormasse und eine Besserung der Symptome. Die Symptome der oberen Einflussstauung zeigen sich meistens in einem Zeitintervall von ein bis zwei Wochen regredient. Die Chemotherapie sollte aufgrund der oben erwähnten Gründe durch eine Vene der unteren Extremitäten appliziert werden. Eine alleinige oder kombinierte Radiotherapie bringt in den meisten Fällen kaum Vorteile und beeinflusst die Langzeitresultate einer Chemotherapie negativ. Ausnahmen sind stark fortgeschrittene kleinzellige Bronchuskarzinome und einige Subtypen der Non-Hodgkin-Lymphome.

Nicht-kleinzellige Lungenkarzinome zeigen sich im Vergleich zu den oben erwähnten Tumoren weniger sensibel auf eine Chemotherapie, weshalb bei diesem Tumortyp die Einlage eines endovaskulären Stents empfohlen wird.

Viele der Malignome, welche zu einem VCSS führen, sind strahlensensibel. Besonders die Bronchuskarzinome zeigen sich in der Folge rasch grössenregredient. Die Radiotherapie ist jedoch kein Garant für einen Therapieerfolg. In ca. 20\% persistieren die Symptome. Es empfiehlt sich daher zunächst einen Stent einzulegen und anschliessend mit der Bestrahlung zu beginnen. [3]

Ein operatives Vorgehen ist bei VCSS kaum indiziert. Eine Ausnahme bildet das Thymuskarzinom, welches unzureichend auf eine Radio- oder Chemotherapie anspricht. Zur besseren Übersicht der Abklärung und Behandlung verweisen wir auf den Diagnose und Therapie Algorithmus adaptiert nach Yu et al. [3]. 
Diagnose und Therapie Algorithmus (Adaptiert nach Yu et al.) [3] Abbildung 1

\section{Prognose}

Im Mittel liegt die Lebenserwartung bei Patienten, welche sich mit einem VCSS in Folge eines Malignoms vorstellen, bei sechs Monaten. Es gibt jedoch eine sehr grosse Spannbreite. In einigen Fällen kann nach einem guten Ansprechen auf eine Chemotherapie eine komplette Remission erzielt werden. Besonders beim nicht-kleinzelligen Lungenkarzinom ist das VCSS jedoch mit einer schlechten Prognose assoziiert. Auch eine Kombination aus Chemo- und Radiotherapie vermag die Langzeitprognose nicht zu verbessern. 


\section{Fallbericht}

Q

\section{Jetziges Leiden:}

Die Zuweisung erfolgte zur weiteren Abklärung einer seit etwa vier Wochen zunehmenden Dyspnoe. Die Patientin beklagte eine intermittierende Atemnot bei zuletzt lediglich leichter Anstrengung. Hinzu kam ein morgendlicher Husten mit gelb-grünlichem Auswurf. Thoraxschmerzen traten nicht auf. Es bestand zudem seit mehreren Wochen ein Schwankschwindel und der Patientin war eine Schwellung im Gesicht, vor allem der Augenlider aufgefallen. Nachtschweiss oder Gewichtsverlust wurden verneint. Im externen Röntgenbild vom Thorax war ein prominenter Hilus rechts ersichtlich.

\section{Systemanamnese:}

Nikotin: 1 Päcken/Tag seit dem 15. Lebenjahr (kumulativ 47 py). Kein Alkohol. Keine B-Symptomatik. Bis auf Östrogenpräparat keine regelmässige Medikation.

\section{Persönliche Anamnese:}

Bland bis auf Varizenstripping vor einigen Jahren.

\section{Familienanamnese:}

Vater 88-jährig an Lungenkrebs verstorben. Mutter 82jährig an Hirntumor verstorben.

\section{Weg zur Diagnose/Kommentar}

Auffallend ist, dass es bei dieser Patientin ohne bisherige Vorerkrankungen zu einer relativ raschen Verschlechterung des Allgemeinbefindens gekommen ist. Wegweisend ist als Leitsymptom die Dyspnoe verbunden mit der neu aufgetretenen Gesichtsschwellung.
Abbildung 2

\section{d}

\section{Status}

62-jährige, wache, allseits orientierte Patientin (GCS 15), normoton und normokard, SpO2 an Raumluft $94 \%$, Atemfrequenz $25 / \mathrm{min}$, afebril, BMI $18.6 \mathrm{~kg} / \mathrm{m} 2$. Gesichtsschwellung mit deutlichen Lidödemen, Plethora im Bereich des Dekolletés. Multiple vergrösserte Lymphknoten supraclavikulär beidseits palpabel, restliche Stationen frei. Halsvenen nicht gestaut. Herztöne rein, keine Nebengeräusche, keine peripheren Ödeme. Bilateral exspiratorisches Brummen über den oberen Lungenfeldern, sonst vesikuläres Atemgeräusch, Orthopnoe und Sprechdyspnoe während Untersuchung. Abdomen weich und indolent, keine Organomegalien, regelrechte Peristaltik, Nierenlogen indolent. Kutane Venenzeichnung im Bereich des Rippenbogens. Digitales Clubbing. Neurologisch bland.

\section{Routinelabor}

Hämoglobin Hämatokrit

\section{Weg zur Diagnose/Kommentar}

Zu beachten ist die Tachypnoe und Orthopnoe. Als klinische Zeichen für eine obere Einflussstauung fallen das Gesichtsödem und die Plethora im Bereich des oberen Thorax auf. Zudem finden sich Kollateralvenen. Das digitale Clubbing könnte im Sinne eines Pierre Marie-BambergerSyndroms als paraneoplastisch gedeutet werden. Vergrösserte supraklavikuläre Lymphknoten sind häufig auf Metastasen (Lunge, Mamma, Magen, Melanom) zurückzuführen.

Abbildung 3 


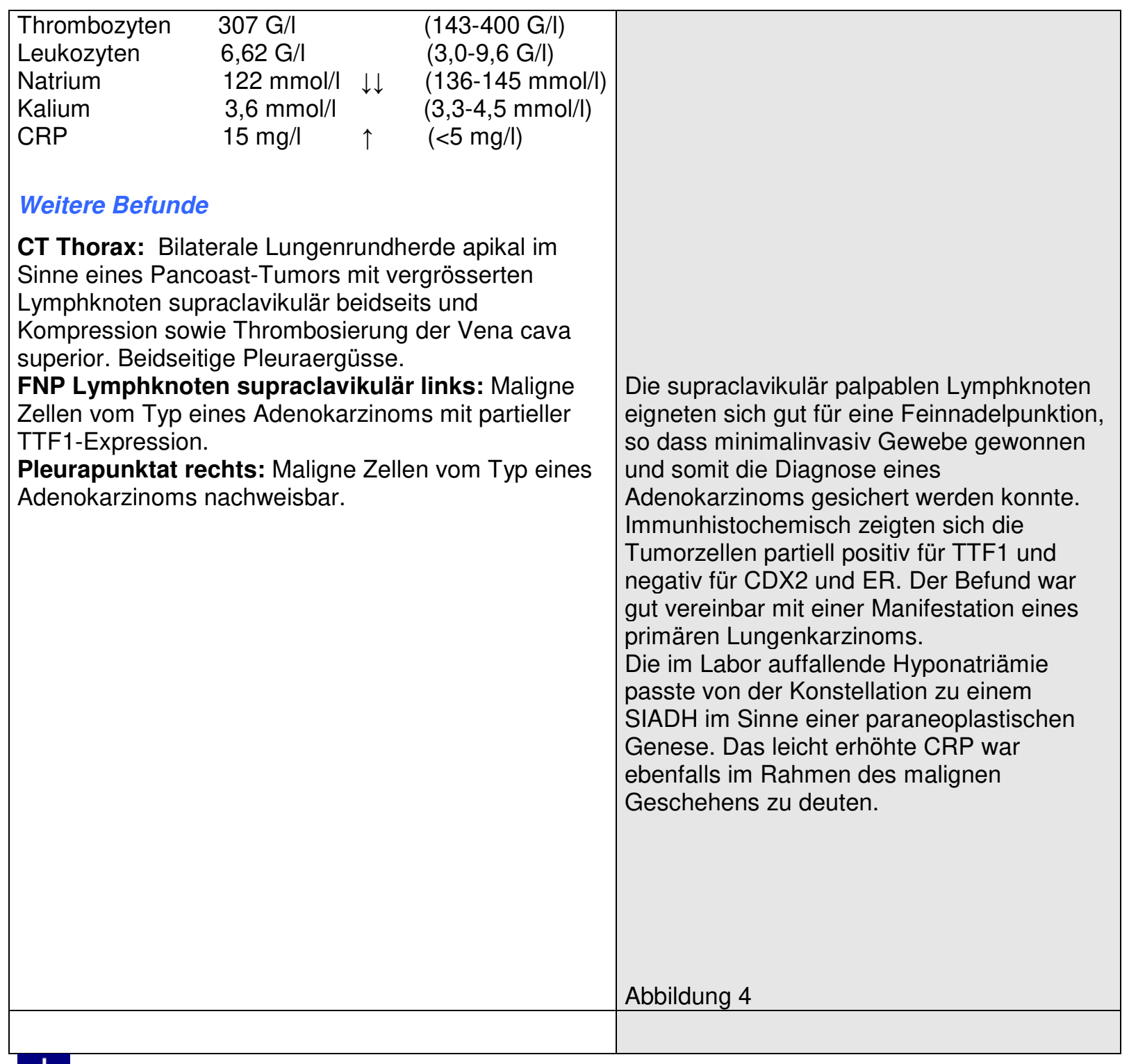

直

Therapie und Verlauf

Bei geplanter Stent-Einlage in die Vena cava superior und bestehender Thrombosierung wurde eine Vollheparinisierung eingeleitet. Die Patientin wurde zur weiteren Behandlung von den Kollegen der Onkologie übernommen. Nach erfolgreicher Wallstent-Einlage zeigten sich die Dyspnoe sowie die Gesichtsschwellung rasch regredient.

\section{Abbildung 5}

Am Folgetag wurde eine palliative Chemotherapie mit Cisplatin und Pemetrexed (Alimta ${ }^{\circledR}$ ) begonnen, welche gut toleriert wurde. Zum Ausschluss von Hirnmetastasen, auch im Hinblick auf den 
persistierenden Schwindel, erfolgte ein MRI vom Schädel. Die hier nachweisbaren kleinen

hämorrhagischen Läsionen im Putamen und frontotemporal links konnten möglicherweise im Rahmen einer thrombo-embolischen Genese beurteilt werden. Metastasen waren aber letztlich nicht ausgeschlossen. Es erfolgte eine Behandlung mit Dexamethason. Bezüglich des Stents wurde eine Antikoagulation mit niedermolekularem Heparin weitergeführt und die Patientin damit nach Hause entlassen.

Leider wurde etwa einen Monat später bei Gangunsicherheit und zunehmender Desorientierung im MRI eine progrediente Hirnmetastasierung deutlich, während bei dem Tumor in der Lunge eine partielle Remission zu sehen war. Eine geplante Ganzhirnbestrahlung sowie jede weitere Therapie wurden von der Patientin abgelehnt. Sie verstarb schliesslich drei Monate nach Diagnosestellung. 


\section{Fragen zur oberen Einflussstauung}

\section{Frage 1}

Welches ist die häufigste Ursache für ein Vena cava superior Syndrom?
a) Thrombus aufgrund von intravasalen Installationen
b) Kleinzelliges Bronchuskarzinom
c) Lymphom
d) Nicht kleinzelliges Bronchuskarzinom
e) Mediastinale Fibrose bei St. n. Syphilis

\section{Frage 2}

Ein Patient wird auf der Notfallstation evaluiert mit Plethora, Stridor und einer beidseitigen Armschwellung. In der Computertomographie lässt sich eine Raumforderung im Bereich der rechten Lungenspitze mit Kompression der Vena cava superior darstellen. Was ist der nächste Schritt?
a) Diagnostische Feinnadelpunktion des Tumors.
b) Notfallbehandlung mittels Stent und Radiotherapie.
c) Therapie mit Kortikosteroiden.
d) Sofortige Chemotherapie mit Carboplatin.
e) Operatives Vorgehen.

\section{Frage 3}

Welches ist der Goldstandart in der Bildgebung für das Vena cava superior Syndrom?
a) Computertomographie
b) Magnetresonanz-Venographie
c) Spiral-CT-Phlebographie
d) Röntgenbild
e) Venographie beider oberen Extremitäten

\section{Frage 4}

Welches ist KEIN klinisches Symptom beim Vena cava superior Syndrom?
a) Kopfschmerzen
b) Produktiver Husten
c) Stridor 
d) Schwellung der oberen Extremitäten

e) Gesichtsplethora

\section{Frage 5}

Welches invasiv-diagnostische Vorgehen weist im Rahmen eines VCSS das höchste Komplikationsrisiko auf?
a) Mediastinoskopie
b) Knochenmarkspunktion
c) Pleurapunktion
d) Lymphknoten-Feinnadelpunktion
e) Bronchoskopie

\section{Autoren}

Dr. med. Harald Nater ${ }^{1}$, Dr. med. Verena Niggemeier ${ }^{1}$, Prof. Dr. med. Rolf. A. Stahel ${ }^{2}$, Dr. med. Daniel Franzen $^{1}$

${ }^{1}$ Klinik und Poliklinik für Innere Medizin, ${ }^{2}$ Klinik für Medizinische Onkologie, Universitätsspital Zürich

\section{Korrespondenzadresse}

Dr. med. Daniel Franzen

Klinik und Poliklinik für Innere Medizin

UniversitätsSpital Zürich

Rämistrasse 100

8091 Zürich

daniel.franzen@usz.ch

\section{Bibliographie}

1. Wilson LD, Detterbeck FC, Yahalom J. Clinical practice. Superior vena cava syndrome with malignant causes. N Engl J Med 2007;356(18):1862-9.

2. Wudel LJ Jr, JC. Superior vena cava syndrome. Curr Treat Options Oncol 2001;2(1):77-91.

3. Yu JB, Wilsen LD, Detterbeck FC. Superior vena cava syndrome--a proposed classification system and algorithm for. J Thorac Oncol 2008;3(8):811 -4

4. Uberoi R. Quality assurance guidelines for superior vena cava stenting in malignant. Cardiovasc Intervent Radiol 2006;29(3):319-22.

5. Rice TW, Rodriguez RM, Light RW. The superior vena cava syndrome: clinical characteristics and evolving etiology. Medicine (Baltimore) 2006;85(1):37-42. 
6. Rowell NP, Gleeson FV. Steroids, radiotherapy, chemotherapy and stents for superior vena caval. Clin Oncol (R Coll Radiol) 2002;14(5):338 51.

7. Urruticoechea A, Mesia R, Dominguez J, et al. Treatment of malignant superior vena cava syndrome by endovascular stent. Lung Cancer 2004;43(2):209 14. 


\section{Antworten zu den Fragen zu XY aus PRAXIS Nr.}

\section{Frage 1}

\section{Richtig ist Antwort d)}

a) Hohlvenenthrombosen aufgrund von intravasalen Installationen sind in Anbetracht von deren Häufigkeit relativ selten.

b) Bei Patienten mit einem kleinzelligen Bronchuskarzinom kommt es aufgrund der raschen Grössenprogredienz häufig zu einem VCSS. Die Inzidenz dieses Tumortyps ist im Vergleich zum nicht-kleinzelligen Bronchuskarzinom jedoch deutlich geringer.

c) Non-Hodgkin Lymphome sind die dritthäufigste Ursache für ein VCSS.

d) RICHTIG.

e) Mediastinale Fibrose bei St. n. Syphilis oder anderen Infektionskrankheiten sind mit dem Zugang zu Antibiotika selten geworden.

\section{Frage 2}

\section{Richtig ist Antwort b)}

a) Bei einem Patienten mit einem Stridor ist eine unverzügliche Behandlung indiziert und die Diagnostik muss aufgeschoben werden.

b) RICHTIG.

c) Eine Therapie mit Kortikosteroiden ist nur indiziert bei etablierter Diagnose eines Lymphoms. Im vorliegenden Fall ist der Tumortyp nicht bekannt.

d) Auch eine Chemotherapie richtet sich nach der Dignität des Tumors und ist in dieser Situation nicht praktikabel.

e) Ein operatives Vorgehen ist selten angezeigt bei Patienten mit einem VCSS aufgrund der geringen Lebenserwartung der Patienten.

\section{Frage 3}

\section{Richtig ist Antwort e)}

a) Mit der Computertomographie mit Kontrastmittel lässt sich das Ausmass der Obstruktion der oberen Hohlvene, Ausprägung und die Anzahl der Kollateralgefässe erkennen.

b) Magnetresonanz-Venographie ist eine Alternative für Patienten mit einer Kontrastmittelallergie oder bei unzureichendem intravenösem Zugang.

c) Spiral-CT-Phlebographie kombiniert die Vorteile der Venographie und CT, ist jedoch anspruchsvoller in der Durchführung.

d) Röntgenbild ist beinahe überall verfügbar und ermöglicht einen ersten diagnostischen Schritt, aber ist meistens unzureichend. 
e) Venographie ist der Goldstandart. Es ist dem CT überlegen zur Abschätzung des Ausmass des VCSS, und die Kollateralgefässe werden dargestellt. Die Aetiologie kann meistens jedoch weniger gut eruiert werden.

\section{Frage 4}

\section{Richtig ist Antwort b)}

a) Im Rahmen der venösen Abflussbehinderung der VCS kann es zum Anstieg des Hirndrucks und damit zu Kopfschmerzen, Schwindel, Synkopen oder Koma kommen.

b) Unproduktiver Husten ist kein direktes Symptom des VCSS, kann jedoch auf ein zugrundeliegendes Bronchuskarzinom hinweisen.

c) Stridor ist die Folge eines Larynxoedems und weist auf eine akute vitale Gefährdung hin.

d) Schwellung der oberen Extremitäten ist eine typische Folge der oberen Einflussstauung.

e) Die Gesichtsplethora gehört zu den milden Symptomen des VCSS.

\section{Frage 5}

\section{Richtig ist Antwort a)}

a) Die Mediastinoskopie weist ein erhöhtes Blutungsrisiko aufgrund des erhöhten venösen Drucks auf. In Hochrisikopatienten ist eine perkutane transthorakale CT-gesteuerte Punktion oder eine endoskopische ultraschallgesteuerte Biopsie eine gute Alternative.

b) Die Knochenmarkspunktion ist eine absolut komplikationsarme Intervention - mit und ohne VCSS. Hat in der Diagnostik bei einem VCSS einen untergeordneten Stellenwert.

c) Bei einer Pleurapunktion kann es gelegentlich zu einem Pneumothorax kommen. Bei sorgfältiger Durchführung ist dieses Risiko jedoch gering und wird durch das Vorhandensein eines VCSS nicht beeinflusst.

d) Feinnadelpunktionen von Lymphknoten sind aufgrund des schmalen Kalibers der Punktionsnadel gefahrlos. Selbst Infektionen und Blutungen sind eine Rarität.

e) Aufgrund des trachealen Ödems im Rahmen der VCSS ist das Risiko von Schleimhautverletzungen und -blutungen beim Vorschieben des Bronchoskopes leicht erhöht. 


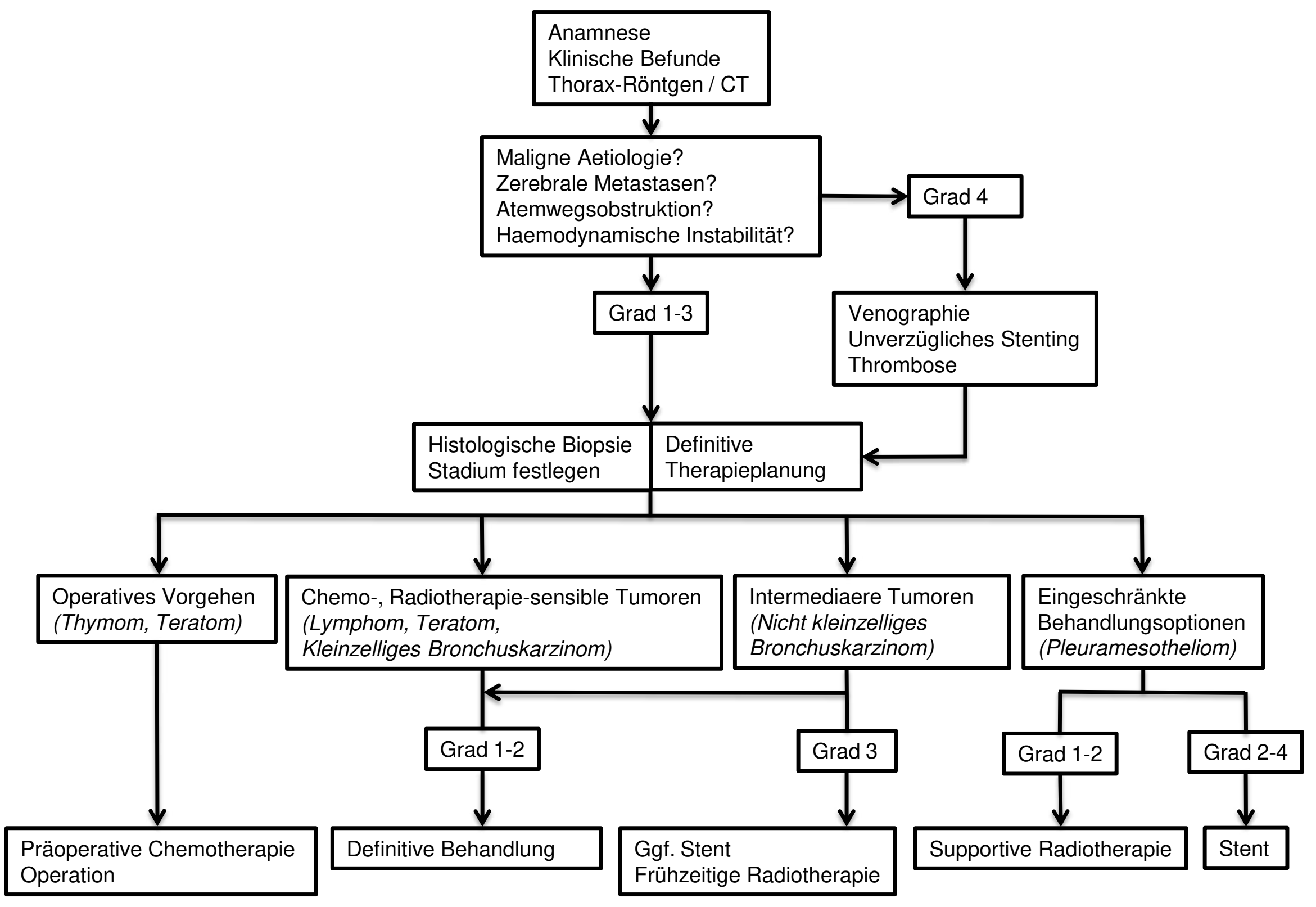




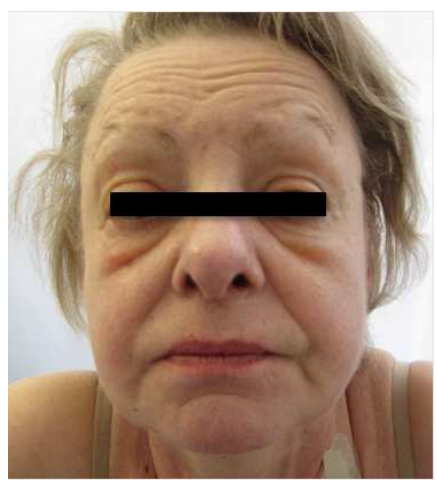




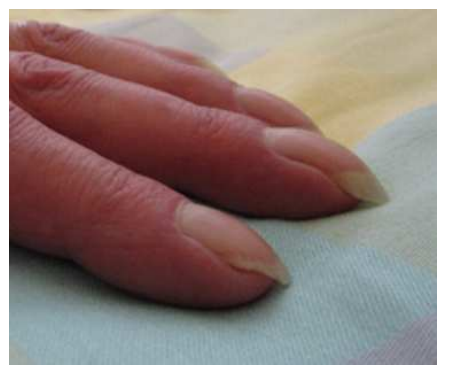





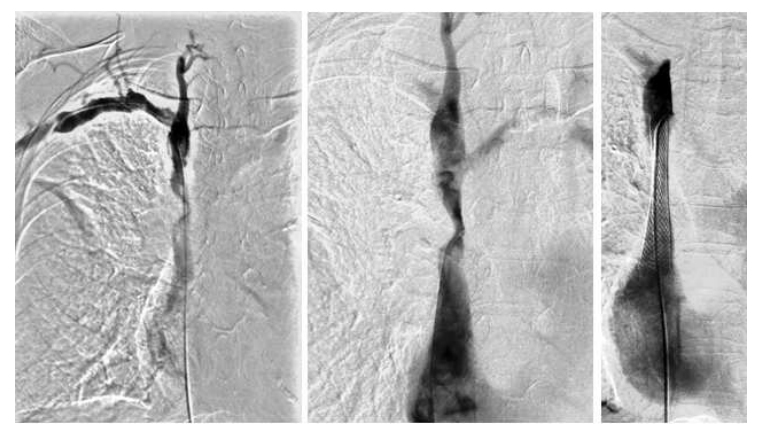

